\title{
De la representación residual de las mujeres en el parlamento portugués al debate parlamentario de la Ley de Paridad (2001): representación mediáti- ca de las diputadas en el debate parlamentario
}

\author{
Ana Cabrera, $\mathrm{M}^{\mathrm{a}}$ Luisa Sánchez Calero, $\mathrm{M}^{\mathrm{a}}$ Lourdes Vinuesa Tejer, \\ Paloma ABEJón MENDOZA* \\ mlusaca@ccinf.ucm.es
}

\author{
(Abstracts y palabras clave al final del artículo) \\ Enviado: 12 de enero de 2012 \\ Evaluado: 10 de abril de 2012 \\ Aceptado: 25 de junio de 2012
}

\begin{abstract}
"Cidadania"
"Não tratamos aqui da igualdade das mulheres e dos homens mas da igualdade de oportunidades para exercer a plena cidadania."

O exercício pleno da cidadania pelas mulheres resulta também aqui numa dupla tarefa: a defesa das suas ideias políticas e a luta por um espaço de intervenção cívica para todos independentemente do sexo.

Só assim se compreende que nos diversos países ocidentais, tal como em Portugal, certos objectivos políticos unam as mulheres para além das ideologias, num combate comum pelo exercício da cidadania."
\end{abstract}

Moção aprovada no Parlamento Paritário em 31 de Janeiro de $1994^{1}$

\section{PRESENTACIÓN Y METODOLOGÍA}

Este artículo se enmarca en el contexto de la investigación que lleva por título, "Política en el femenino: políticas de género y estrategias de visibilidad de las diputadas parlamentarias en Portugal" "2. Es un proyecto financiado por la Fundación para la Ciencia y Tecnología de Portugal, que seguimos desarrollando desde 2010 en El Centro de Investigación Media e Jornalismo (CIMJ)3).

* Ana Cabrera es Investigadora do Centro de Investigação Media e Jornalismo y Profesora del Departamento de Ciencias de Comunicación de la Universidade Nova de Lisboa, María Luisa Sánchez Calero es Profesora del Departamento de Periodismo I de la Facultad de Ciencias de la Información de la UCM, Maria Lourdes Vinuesa es Profesora del Departamento de Sociología VI de la Facultad de Ciencias de la Información de la UCM, y Paloma Abejón es Profesora del Departamento de periodismo I de la Facultad de Ciencias de la Información de la UCM.

${ }^{1}$ Esta moción ha sido aprobada en el Parlamento Paritario ante la iniciativa de tres eurodiputadas. En Enero de 1994 el mismo número de diputadas y diputados se reunieron en la Sala del Senado del Parlamento con el objetivo de debatir el déficit de la participación femenina en la política.

2 Este artículo se enmarca en el proyecto de investigación "Política en el femenino: políticas de género y estrategias de visibilidad de las diputadas parlamentarias", financiado por la Fundación para la Ciencia y Tecnología de Portugal.

${ }^{3}$ En Portugal la investigación se organiza en torno a varias Unidades de Investigación cuyo trabajo se desarrolla en un área del saber. El Centro de Investigación Media y Periodismo es un centro autónomo asociado a la Facultad de Ciencias Sociales y Humanas de la Universidad Nova de Lisboa. 
El proyecto se centra en la representación de las diputadas y las cuestiones de género en el Parlamento portugués a lo largo de tres ciclos políticos de la democracia portuguesa desde 1976 hasta 2002: El periodo del Proceso Revolucionario en Curso (PREC) entre 1975-76; El Bloco Central (1983-1985); El Cavaquismo que corresponde a la mayoría parlamentaria del Partido Social Demócrata (PPD/PSD), periodo de los gobiernos de Cavaco Silva (1985-1991); El Guterrismo, que corresponde al periodo en el que Antonio Guterres, líder del Partido Socialista (19952002) ganó las elecciones. Para cada uno de los ciclos políticos hemos seleccionado los acontecimientos de género. En otras palabras, se trata de resaltar aquellas cuestiones debatidas en el Parlamento que hablan sobre la igualdad entre hombres y mujeres y que simultáneamente han tenido cobertura en los medios de comunicación: la cuestión de los derechos e igualdad, el debate de la ley del Aborto (que ocupa dos ciclos políticos); el Parlamento Paritario y la ley de la Paridad (dos ciclos políticos).

La metodología común en toda la investigación se centra en un análisis cuantitativo y cualitativo con el objeto de conocer quiénes son las diputadas en el periodo desarrollado entre el año 1976 y 2002. Para ello, hemos elaborado una base de datos en el programa informático SPSS que nos ha permitido trazar el perfil de las diputadas en cada ciclo político: número de mujeres electas, edad, profesión, formación académica, su pertenencia en las diferentes Comisiones o sus intervenciones en el plenario, entre otras.

Por otra parte, se realiza un análisis de prensa en diferentes medios impresos con diferentes líneas editoriales que nos permitirá conocer la representación que alcanza la mujer parlamentaria en los cinco periódicos seleccionados para la muestra. Estos periódicos representan los cambios experimentados durante 27 años que son los que recoge esta investigación para su análisis. Como muestra para este artículo sobre La ley de la Paridad, hemos seleccionado dos semanarios y tres diarios. Entre los semanarios se encuentran el Expresso que está representado en todo el periodo de la investigación. Se trata de un periódico de referencia, conocido por su calidad periodística, equilibrio y clara separación entre la información y la opinión. $O$ Independente es un semanario más especulativo, con una gran relación con el partido de Centro Democrático Social- Partido popular (CDS/PP). Desde luego por su director Paulo Portas que actualmente es el líder de este partido y miembro del actual gobierno. Durante su existencia este semanario no dejó de denunciar casos en que estaban envueltos ministros del gobierno de Cavaco Silva. El Correio da Manhã es un periódico con una línea más popular: pequeñas noticias, asuntos sociales, especulaciones y cuestiones familiares. Por otro lado, los otros dos diarios considerados de referencia son: Público; un periódico destinado a las elites intelectuales y el Diario de Noticias el periódico portugués más antiguo que sigue publicando desde 1864.

En base a cada acontecimiento se han seleccionado las diferentes informaciones en los periódicos mencionados. Todas las piezas han sido introducidas en una base de datos que nos han permitido calcular el número de informaciones por periódico, los títulos y las fechas, el género periodístico, la sección y número de página que ocupa, y los protagonistas de la información que intervienen en las piezas periodísticas. Finalmente se procede al análisis complementándolo con declaraciones y tes- 
timonios procedentes de las diputadas y que aparecen reflejados igualmente en un destacado de la información.

Pero en este artículo nos ceñiremos exclusivamente a representar las conclusiones extraídas del análisis que hacen referencia al debate parlamentario sobre la Ley de la Paridad. Una ley que ha sido debatida en el ciclo político del Guterrismo en el año 2002. En este caso los objetivos marcados estarán centrados en: comprender el contexto internacional y nacional del debate de la Ley de la Paridad; conocer a las diputadas parlamentarias en el periodo del Guterrismo y analizar finalmente la representación que alcanzan las diputadas en la prensa durante el debate de la Ley de la Paridad.

Así pues, primero nos detendremos en los marcos teóricos más significativos para este trabajo con el objeto de explicar posteriormente el contexto internacional y nacional de las cuestiones de la paridad y de las cuotas. Seguidamente explicaremos la situación de las diputadas parlamentarias en la $8^{\text {a }}$ Legislatura (1999-2002)

\section{EL MARCO TEÓRICO}

En esta investigación el concepto de género abarca tanto el femenino como el masculino y se considera un diferencial de poder político entre mujeres y hombres. En los estudios de género la literatura se ha centrado en la importancia de la representación equitativa y en las opiniones de los expertos que defienden argumentos simbólicos y los que valoran argumentos sustantivos como han señalado Norris e Lovenduski (1989).

Así pues los partidarios de argumentos simbólicos defienden que un parlamento, como fórum público, será democrático desde que incluye puntos de vista diversos y distintos. Mientras que aquellos otros que defienden argumentos sustantivos evidencian y demuestran que las mujeres son portadoras de perspectivas únicas en valores y actitudes que lo proyectan como una de sus principales prioridades políticas y que tienen efectos en las Leyes y en las decisiones.

Pero una cantidad razonable de la investigación se detiene, no en las ventajas de la participación equitativa de las mujeres en cargos políticos, sino más bien en los obstáculos que las mujeres deben superar para ser elegidas, lo que nos reconduce a la cuestión de las cuotas (Ballington e Karam 2005; Sawer, Marian et al. 2006; Paxton e Hughes 2007; Norris e Inglehart 2001).

Los autores están de acuerdo que en política las mujeres están sub-representadas y que esta discrepancia se ha transformado por motivos de justicia y equidad en un poderoso argumento a favor de la paridad de género. Pero la cuestión de las cuotas sigue siendo para la sociedad un tema de divergencia. Sobre este asunto Silveirinha y Peixinho (2008) comenta que si por un lado las cuotas pueden ser encaradas como una forma de discriminación positiva, sobre todo en los países que tienen una reducida participación de mujeres en la política; otros consideran esta situación como indeseable. En su estudio estas autoras han concluido que "las cuotas siguen siendo polémicas y no hay garantías de que, efectivamente, proporcionen una mejor demo- 
cracia. En todo caso, una mejor democracia donde las identidades que conforman nuestra ciudadanía son reconocidas, no solo en los procesos formales de representación, sino en las opiniones que circulan en la esfera pública, que legitiman las normas acordadas sobre la verdadera igualdad entre mujeres y hombres" (Silveirinha y Peixinho, 2008: 12)

Además, hay argumentos suficientes, como destaca Phillips (1998:239) para abogar por la presencia de un mayor número de mujeres en la política y que sirvan de base a un cambio sustancial. Asimismo, el tema de la paridad de género en la política ha sido estudiado por diversos expertos, sobre todo como enfoque entre las diversas situaciones y experiencias en varios países del mundo (Norris, 2000; Lovecy, 2002; Sgier, 2003; Ross, 2003; Amâncio, y Santos 2004; Krook 2006; Galligan, 2006; Zetterberg, 2009)

Por ello estudiar la representación que alcanza la mujer parlamentaria en la prensa equivale a analizar como proyectan los perfiles en ella y como crean prototipos en la sociedad que terminan construyendo estereotipos. En verdad es en base a esta representación mediática cuando los ciudadanos construyen sus opiniones. Es decir que "la representación constituye la selección o exclusión de ciertos elementos de la realidad, para construir una nueva realidad", en la que se excluyen unos elementos y se seleccionan otros (Almeida, 1986:493). Para Karen Ross (2003) si se considera a la noticia como una mercancía y todos somos consumidores, como tal será importante analizar cuantas veces aparecen las mujeres políticas en la prensa y lo que es aún más importante comprender en qué contexto aparecen ellas.

Dos estudios recientes en España sobre el tema han demostrado el avance significativo que ha alcanzado la mujer parlamentaria en los medios de comunicación. Para Vinuesa Tejero,L; Abejón Mendoza, P y Sánchez Calero, Mª L. (2011) existe entre Mujeres y Política un binomio con baja representación en los medios de comunicación españoles. Esto también lo confirman los datos representados por Vilatod i Presas (2008) cuando advierten sobre la construcción de la imagen de las mujeres políticas en los medios de comunicación.

\section{LA MUJER PORTUGUESA Y SU PARTICIPACIÓN EN LA POLÍTICA}

Muchos aspectos han participado en el cambio favorable que se ha alcanzado en la situación de desigualdad sufrida anteriormente en la representación política de género. Pero el marco principal es sin duda el 25 de Abril con la institucionalización de la democracia. Además la libertad y la democracia han posibilitado la aparición de varios movimientos de mujeres y la influencia de los programas internacionales de la ONU y la Unión Europea.

Por otro lado en Portugal existen diferentes corrientes ideológicas, conducidas por diversos movimientos feministas. Pero a pesar de las diferencias existentes en estas organizaciones de mujeres, todas han contribuido en denunciar cuestiones relacionadas con las formas de discriminación y han contribuido en programar acciones 
contra la desigualdad en materia política económica y social ${ }^{4}$. La acción de la "Comisión de la Condición Femenina"5 está presente en su publicación oficial ${ }^{6}$ y proporciona una importante información para identificar varios de los episodios de la lucha de las mujeres por la igualdad.

Ana Vicente ${ }^{7}$ (1997: 8-10) considera que la Comisión para la Igualdad de Derechos de las Mujeres (CIDM), actúa como vanguardia de los movimientos de mujeres en Portugal. Su lenguaje es muy reivindicativo: "Apoyar todas las formas de sensibilización de las mujeres portuguesas y la eliminación de las discriminaciones por formar u procesar su integración en la sociedad portuguesa en consonancia con los principios constitucionales".

Sin embargo, Manuela Tavares (2010: 383) cree que los años comprendidos entre 1980 y 1990 se caracterizaron por una menor movilización más que por una acción sistemática de las organizaciones internacionales especialmente la de las Naciones Unidas. En este sentido la autora sostiene que en la década de los 90 los movimientos feministas se globalizan. Por eso la cuestión de la Paridad en el Parlamento surge en un contexto internacional de valoración de la participación política de las mujeres. En este sentido las iniciativas de la ONU entre las que se encuentra la Conferencia de Mujeres de las Naciones Unidas sobre el tema Igualdad en la Participación Política creada en 1985 en Nairobi han contribuido en ello . En esta conferencia se aprobó, para los quince años siguientes el estatuto de la mujer para el tema de Igualdad, Desarrollo y Paz.

Como consecuencia a la integración de Portugal como miembro de la Comunidad Europea en 1986, el gobierno incorporó en las leyes nacionales las directivas europeas sobre igualdad. La idea de democracia paritaria ha sido introducida por el Parlamento Europeo considerando que la democracia implicaba la igualdad entre mujeres y hombres en cargos políticos. Por otro lado, las conclusiones de la Conferencia de Pekín (1995) han difundido la idea de poder de las mujeres en el sentido de liderazgo. Así se ha producido un cambio estructural en la forma de ver a la mujer: cesa el proceso de victimización para dar lugar a la afirmación de su poder, de su autoconfianza, de su responsabilidad y de su liderazgo.

Sin embargo, el trabajo y el ejercicio de la vida política siguen siendo mayoritariamente masculinos. En realidad una democracia se transforma en un régimen frágil si excluye a las mujeres de la vida política. A este propósito, Cabo (2002) sustenta la paridad como proceso estratégico de lucha contra el monopolio masculino en el poder. Considera también que la democracia paritaria nace de la contradicción existente entre el aumento de la participación femenina en diversos sectores de la

4 Entre las diversas organizaciones feministas destacamos: Movimento de Libertação das Mulheres (MLM); Movimento Democrático de Mulheres (MDM), União das Mulheres Alternativa e Resposta (UMAR); GRAAL.

5 Comisión de la Condición Femenina fundada en 1970. Es una organización gubernamental. Después del 25 de Abril desarrollaron una dinámica para alertar a la situación de las mujeres sobre las cuestiones de desigualdad. Esta Comisión pasa a partir de 1991 a ser denominada como Comisión para la Igualdad de Derechos de las Mujeres (CIDM) y a partir de Mayo de 2007 a ser designada como la Comisión para la Ciudadanía e Igualdad de Género (CIG)

6 "Noticias" Archivo de la Comisión para la Ciudadanía e Igualdad de Género. 
vida social y su ausencia en los espacios donde se votan las leyes que afecta a toda la sociedad y en particular a las mujeres (Cabo, 2003:31)

\section{EL GUTERRISMO (1995-2002) Y SU DISCURSO INCLUSIVO}

Partimos de la idea de que una democracia donde la mitad de sus ciudadanos son excluidos de la participación política es una democracia frágil. Cabo (2002:31) defiende la paridad como un proceso estratégico de lucha contra el monopolio masculino en el poder. En Portugal algunos años antes del primer debate parlamentario sobre la ley de la paridad, tres eurodiputadas, María Belo (Partido Socialista - PS), Margarida Salema (Partido Social demócrata - PSD) e María Santos (Partido Ecologista os Verdes coligado con o Partido Comunista Portugués - PEV) propusieron la iniciativa del Parlamento Paritario. Durante dos días (31 de Enero y 1 de Febrero) el mismo número de diputadas y diputados se reunieron en la Sala del Senado del Parlamento con el objetivo de debatir el déficit de la participación femenina en la política.

La iniciativa resultó todo un éxito desde el punto de vista de la cobertura informativa por ser un seudo-acontecimiento (Boorstin 1962). Fueron aprobados dos documentos: "La Ciudadanía" y la "Carta para una participación política paritaria". Ambos tuvieron efectos importantes en el ciclo político siguiente con Antonio Guterres (líder del Partido Socialista). Este, como principal líder de la oposición mostró una particular sensibilidad en abrazar la causa de la paridad, ante todo por haber estado presente en los días de la iniciativa y además por haber anunciado la presentación de un proyecto de ley para aplicar en las listas electorales la proporción de una mujer por cada cuatro hombres. La mayoría obtenida por el Partido Socialista en las elecciones de 1995 puso fin al ciclo político del Cavaquismo cuyo primer ministro Cavaco Silva y la mayoría del Partido Social Demócrata puso fin a su mandato iniciándose la siguiente etapa del Guterrismo.

Por primera vez se presenta una etapa donde el primer ministro utiliza un lenguaje no sexista que ya lo había aplicado como líder de la oposición y durante la campaña electoral. Esta actitud anticipa la futura revisión que será consagrada en el Tratado de Maastricht sobre la sustitución del lenguaje sexualmente neutral para un lenguaje no sexista. Este hecho demostró la atención que el nuevo primer ministro intentaba proporcionar a las cuestiones derivadas de la igualdad de género.

En la sesión del Consejo Consultivo de la Comisión para la Igualdad de Derechos de la Mujer, celebrada el 7 de Marzo de 1996, el primer ministro Antonio Guterres dijo, "Hay diferencia entre el país real y el país legal y la igualdad aunque consagrada en la ley no es siempre respectada en la práctica social" 8 .

Guterres era favorable a la discriminación positiva y lo demuestra defendiendo que "a través de la ley y de la acción será posible extinguir la "enfermedad" de las

\footnotetext{
7 Presidente de la Comisión para la Igualdad y de Derechos de las Mujeres (CIDM) entre 1992-96.

8 "Notícias", n. ${ }^{\circ} 38, \mathrm{Jan} / \mathrm{Fev} / \mathrm{Mar}$ de 1996, p 31. Archivo de la Comisión para la Ciudadanía e Igualdad de Género.
} 
sociedades mediterráneas que es el predominio efectivo de los hombres en la esfera pública". ${ }^{9}$ Incluso presentó nuevas medidas en torno a la cuestión de género: protección a las mujeres víctimas de violencia doméstica, ejercicio de la maternidad o de la paternidad; leyes de trabajo. Guterres pensaba que era necesario ir más allá de vigilar y hacer cumplir las leyes. Por tanto crea un Plan Global para la Igualdad y el gobierno adopta también las propuestas de la Plataforma de Pekín del $4^{\circ}$ Programa de Acción Comunitaria. ${ }^{10}$

En 1995 el Parlamento introduce una Comisión Parlamentaria para la Paridad e igualdad de oportunidades, presidida por Rosario Carneiro. Esta diputada explica que la comisión fue una iniciativa del Partido Socialista y de la diputada Helena Roseta para "luchar por la cuestión de la paridad y de igualdad de oportunidades entre mujeres y hombres en el Parlamento". 11

Concluiremos por tanto que este nuevo ciclo político estuvo marcado por el interés en las cuestiones de igualdad de género y por la paridad en el sistema electoral. Así existía en Portugal una voluntad política favorable al debate de la Paridad. El gobierno tomó la delantera al presentar en la Asamblea de la Republica la Ley, mientras que la preparación y coordinación del debate parlamentario quedó a cargo de María de Belém Roseira. Esta diputada y antigua Ministra de Salud y, más recientemente Ministra de Igualdad, preparó como diputada el debate en la Asamblea de la Republica. Antes del día 30, fecha marcada para la votación, María de Belém presidió las conversaciones teniendo como objetivo un consenso entre los partidos sobre todo con el Bloco de Esquerda. Esta diputada seguía estos principios: "Si el programa político es visto y analizado por una sola parte de la humanidad, eso no es favorable para la democracia representativa. No tiene sentido perder talentos desconocidos e ignorados". (Público, 29/03/2002). A pesar de los esfuerzos y conversaciones la ley no fue aprobada y contó con la oposición de dos partidos: Partido Social Demócrata y Partido Comunista. Manuela Ferriera Leite diputada del Partido Social Demócrata, mujer con larga experiencia en política, fue Secretaria de Estado y Ministra y defendió que "Con este sistema las mujeres serían observadas, no por su mérito, formación o competencia, sino porque obligatoriamente tenían que estar alli para hacer número... Es humillante... se está luchando no por un derecho, que ya está recogido en la ley. Esto es una cuestión cultural que no se soluciona por decreto." (Público, 29/03/2002). También Margarida Tengarrinha diputada por el Partido Comunista demuestra su oposición a la Ley de la Paridad acentuando la inconfundible marca de su posición ideológica: "Considero la ley como una farsa porque la ausencia de mujeres en los centros de decisiones políticas está inequívocamente asociada a la discriminación económica, social y cultural sufrida por la mujer." (Público, 29/03/2002)

\footnotetext{
9 "Notícias", n. . 38, Jan/Fev/Mar de 1996, p 31. Archivo de la Comisión para la Ciudadanía e Igualdad de Género.

10 "Noticias", n. ${ }^{\circ}$ 38, Enero/Febr/Mar de 1996, p 31. Archivo de la Comisión para la Ciudadanía e Igualdad de Género.

${ }^{11}$ Entrevista con María del Rosario Carneiro en el diario "Noticias", n. ${ }^{\circ} 41$, Abr/May/Jun de 1997, p 33. Archivo de la Comisión para la Ciudadanía e Igualdad de Género.
} 


\section{EL PERFIL DE LAS DipUtAdAS EN EL PARLAMENTO PORTUGUÉS DURANTE EL GUTERRISMO}

Los resultados que presentamos están basados en varias fuentes documentales procedentes del Archivo Histórico de la Asamblea Parlamentaria de la República como son los Registros Individuales de los Diputados; las Actas de presencia de las diputadas en las comisiones; el "Diario Asamblea de la República” y las Bases de Datos de la Asamblea de la Republica: PLC, Gode, que se trasladaron a una base de datos soportada en el sistema estadístico SPSS ${ }^{12}$. Además, los outputs que presentamos están basados en los Registros Individuales de los Diputados lo que nos permitió conocer la identificación de los mismos y los movimientos de entradas y salidas en el Parlamento.

El plenario de la Asamblea de la República está constituido por 230 diputados. Sin embargo el número de diputadas registradas es siempre mayor debido al constante movimiento de entradas y salidas que ellas realizan en el Parlamento.

No obstante, la metodología seguida por los servicios del Parlamento es diferente en el recuento de los diputados que aquella realizada en el primer día de funcionamiento del plenario en la Asamblea de la República.

Se observa como en esta legislatura el número de diputadas aumentó y casi se duplicó en relación al ciclo político anterior. Sin embargo, aún notamos una enorme diferencia en la representación de 61 diputadas frente a 268 diputados, como se observa en el gráfico 1 que representa el total de diputadas y diputados por partido político según los registros biográficos obtenidos.

Gráfico $\mathrm{N}^{\circ}$ 1. -Datos relativos a los registros biográficos de las diputadas y de los diputados en la Asamblea de la República entre 1999-2002

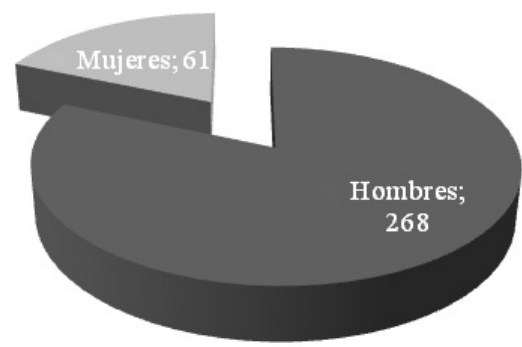

Sin embargo en el cuadro $\mathrm{n}^{\mathrm{o}} 1$ se observa el total de diputadas y diputados por partido político en la $8^{\mathrm{a}}$ Legislatura (1999-2002). Según el registro biográfico, el

12 SPSS- Statistical Package for the Social Sciences 
Partido Socialista (PS) tiene un total de 170 diputados de los cuales 129 son hombres $(39,21 \%)$ y 41 son mujeres $(12,46 \%)$. Este representa al partido con mayor número de diputadas en el Parlamento. EL Partido Social Demócrata (PPD/PSD) con un total de 105 diputados en el Parlamento, donde 94 son hombres $(28,57 \%)$ y 11 son mujeres $(3,34 \%)$. Le sigue el Partido Comunista Portugués (PCP) con un total de 19 diputados donde 14 (4,26\%) son hombres y solo 5 (1,52\%) son mujeres. El Partido Ecologista de los Verdes (PEV) es la única organización política que alcanzó el mismo número de diputadas que de diputados con un total de 4 representantes. El Centro Democrático Social (CDS) alcanzó de 27 parlamentarios y tan solo una solo diputada. El Bloco de Esquerda (BE) obtuvo 4 representantes y entre ellos solo una es mujer.

Cuadro $\mathrm{n}^{\mathrm{o}} 1$ - Total de diputadas y diputados por partido político en la $8^{\mathrm{a}}$ Legislatura (19992002). Segundo registro biográfico.

\begin{tabular}{|c|c|c|c|c|c|c|}
\hline & \multicolumn{2}{|c|}{ N. ${ }^{0}$ de Hombres } & \multicolumn{2}{|c|}{ N. ${ }^{\circ}$ de Mujeres } & \multicolumn{2}{|c|}{$\begin{array}{c}\text { Total de } \\
\text { deputados }\end{array}$} \\
\hline & $\begin{array}{l}\text { N. }{ }^{\circ} \\
\text { Dep }\end{array}$ & $\%$ & $\begin{array}{l}\text { N. } \\
\text { Dep }\end{array}$ & $\%$ & $\begin{array}{l}\text { N. }{ }^{\circ} \\
\text { Dep }\end{array}$ & $\%$ \\
\hline $\mathbf{B E}$ & 3 & 0,91 & 1 & 0,30 & 4 & 1,22 \\
\hline CDS & 26 & 7,90 & 1 & 0,30 & 27 & 8,21 \\
\hline PCP & 14 & 4,26 & 5 & 1,52 & 19 & 5,78 \\
\hline PEV & 2 & 0,61 & 2 & 0,61 & 4 & 1,22 \\
\hline PPD/PSD & 94 & 28,57 & 11 & 3,34 & 105 & 31,91 \\
\hline PS & 129 & 39,21 & 41 & 12,46 & 170 & 51,67 \\
\hline Total & 268 & 81,46 & 61 & 18,54 & 329 & 100 \\
\hline
\end{tabular}

Lo que nos demuestra que aquellos partidos con representación mayoritaria siguen teniendo un mayor número de diputadas puesto que las colocan en el medio de las listas. Se puede observar como en función de los resultados electorales son los partidos ganadores a quienes se les atribuyen un mayor número de escaños femeninos, en cambio aquellos que pierden se quedan con menos diputadas.

A este respecto, Araujo (2005:195) señala que en la selección de los diputados se tiende a desarrollar una lógica que es, a la vez inercia y pragmática y, por supuesto se reproduce de una manera más intensa en los sistemas mayoritarios. Se supone que los candidatos que ya están en el parlamento o que han tenido buenas votaciones en anteriores elecciones, tienden a ofrecer menos riesgo en la competición, porque su nombre es conocido, y tienen apoyos y una estructura organizada. Por lo tanto, una parte significativa de las prioridades tiende a dirigirse hacia aquellos que ya están ocupando posiciones, o que están tratando su re-elección. Los que compiten por primera vez, se les seleccionan de acuerdo a un perfil más tradicional y semejante al de los hombres. 
Cuadro $\mathrm{n}^{\mathrm{0}} 2$ - Mandato de las diputadas por partido político. $8^{\mathrm{a}}$ Legislatura (1999-2002)

\begin{tabular}{|c|c|c|c|c|c|c|c|}
\hline \multirow{2}{*}{ GP/Mandato } & $\mathbf{B E}$ & CDS & PCP & PEV & PPD/PSD & PS & Total \\
\hline & N. ${ }^{\circ}$ Dep & N. ${ }^{\circ}$ Dep & N. ${ }^{\circ}$ Dep & N. ${ }^{\circ}$ Dep & N. ${ }^{\circ}$ Dep & N. ${ }^{\circ}$ Dep & N. ${ }^{\circ}$ Dep \\
\hline $\begin{array}{c}\text { Electas y } \\
\text { ocupando } \\
\text { sus cargos }\end{array}$ & - & 1 & 3 & 2 & 9 & 19 & 34 \\
\hline Sustituidas & 1 & - & 2 & - & 2 & 22 & 27 \\
\hline Total & 1 & 1 & 5 & 2 & 11 & 41 & 61 \\
\hline
\end{tabular}

El cuadro $n^{\circ} 2$ representa al mandato de las diputadas que son 61 inscritas. Entre ellas han sido 27 las diputadas reemplazadas durante la legislatura: El Partido Socialista ha sustituido a más de 22 diputadas mientras el Partido Social Demócrata y Partido Comunista sustituyeron respectivamente a dos de sus diputadas, sin embargo en el Bloco de Esquerda no se produjeron sustituciones. Lo que demuestra que en esta $8^{\text {a }}$ Legislatura (1999-2002) sean 34 las diputadas que ocupen el cargo.

Cuadro n³ - Formación académica de las diputadas

\begin{tabular}{ccc}
\hline Formación Académica & $\begin{array}{c}\mathbf{N .}^{\mathbf{0}} \\
\text { Dep }\end{array}$ & $\mathbf{\%}$ \\
\hline $\begin{array}{c}\text { Formación en estudios } \\
\text { secundarios }\end{array}$ & 1 & 1,64 \\
$\begin{array}{c}\text { Finalizados los estudios en } \\
\text { secundaria }\end{array}$ & 2 & 3,28 \\
$\begin{array}{c}\text { Formación en estudios } \\
\text { universitarios }\end{array}$ & 5 & 8,20 \\
Finalizada su Licenciatura & 43 & 70,49 \\
Estudios de post-graduados & 5 & 8,20 \\
NA / ND & 5 & 8,20 \\
Total & $\mathbf{6 1}$ & $\mathbf{1 0 0}$ \\
\hline
\end{tabular}

En el cuadro $\mathrm{n}^{\circ} 3$ se observa la formación académica de las diputadas y se percibe que un total de 43 diputadas tienen alguna formación académica lo que corresponde a un $70,49 \%$ de la muestra. Del resto solo cinco de estas diputadas poseen una formación en estudios universitarios $(8,20 \%)$ y otras cinco tienen estudios de postgraduados $(8,20 \%)$. Además el análisis sobre la actividad profesional de las diputadas demuestra que ellas ocupaban mayoritariamente cargos en profesiones intelectuales y científicas. 


\section{REPRESENTACIÓN DE LAS MUJERES PARLAMENTARIAS EN LA PRENSA DURANTE EL DEBATE DE LA LEY DE PARIDAD (2001)}

El debate ha sido rápido en el Parlamento como consecuencia de que en anteriores negociaciones entre los líderes partidarios han demostrado que la ley no sería aprobada. Sin embargo, el debate parlamentario sobre la cuestión de las Cuotas ha generado 14 informes en la prensa, como demuestran los datos representados en el Cuadro ${ }^{\circ}$ 4, que marca el periodo entre el 24 de Marzo y 6 de Mayo de 2001.

El periódico Público se adelanta con 6 informaciones $42,86 \%$, el semanario Expresso presenta 4 piezas informativas 28,57\% y Correio da Manhã, un periódico popular de gran circulación, no ha elegido ninguna información.

Cuadro $\mathrm{n}^{\mathrm{0}} 4$ - Selección de informaciones entre el 24 de Marzo y 6 de Mayo

\begin{tabular}{ccc}
\hline Publicación & N. $^{\boldsymbol{o}^{2}}$ & $\mathbf{\%}$ \\
\hline Correio da Manhã & - & - \\
Diário de Notícias & 2 & 14,29 \\
Público & 6 & 42,86 \\
Independente & 2 & 14,29 \\
Expresso & 4 & 28,57 \\
Total & 14 & 100 \\
\hline
\end{tabular}

La mayoría de las informaciones publicadas se redactan como Comentario/Opinión/Crítica/Crónica, como se muestra en el cuadro ${ }^{\circ} 5$. En realidad lo que demuestra este dato es que aún no existía consenso sobre el tema porque se encontraba en una fase de debate, sobre todo si añadimos el dato de que la cobertura periodística había sido escasa.

Cuadro $\mathrm{n}^{\circ} 5$ - Género periodístico dominante

\begin{tabular}{ccc}
\hline Género Periodístico & N. $^{{ }^{\mathbf{0}}}$ & $\mathbf{\%}$ \\
\hline Noticia & 4 & 28,57 \\
Reportaje & 1 & 7,14 \\
Entrevista & 1 & 7,14 \\
Comentario/Opinión/Crítica/Crónica & 6 & 42,86 \\
Editorial & 1 & 7,14 \\
Testimonio & 1 & 7,14 \\
Total & 14 & 100 \\
\hline
\end{tabular}

Se observa como el periódico Público es quien ha publicado un mayor número de informaciones en sus páginas y también se le atribuye la mayor diversidad de géneros periodísticos: dos noticias, un reportaje, una crónica, un editorial y diversos testimonios. 
Sin embargo, el análisis de la ubicación de informaciones sobre la ley de la Paridad demuestra que la mayoría de las informaciones no han tenido presencia en la prensa y que solamente el periódico Público lo publica en primera página.

Cuadro $\mathrm{N}^{\mathrm{o}} 6$ - Ubicación de informaciones por publicación

\begin{tabular}{|c|c|c|c|c|c|c|}
\hline Ubicación de las informaciones & Correio da Manhã & $\begin{array}{c}\text { Diario de } \\
\text { Noticias }\end{array}$ & Público & Independente & Expresso & Total \\
\hline Llamada de $1^{\mathrm{a}}$ página & - & - & 2 & - & - & 2 \\
\hline Un destaque de $1^{\mathrm{a}}$ página & - & 2 & 4 & 2 & 4 & 12 \\
\hline Total & - & 2 & 6 & 2 & 4 & 14 \\
\hline
\end{tabular}

También es el periódico Público el que mayor número de páginas ha concentrado sobre el asunto del debate de la Ley de la Paridad y quien más fotos ha editado sobre el tema. No podemos dejar de subrayar que este trabajo periodístico ha sido organizado por una periodista con larga experiencia e interés en acompañar temas de género ( São José Almeida).

Cuadro $\mathrm{n}^{\circ} 7-$ Total de actores en las informaciones periodísticas

\begin{tabular}{cccc}
\hline Actor/Género & Hombres & Mujeres & Total \\
\hline Actor principal & 7 & 20 & 27 \\
Actor secundario & 6 & 12 & 18 \\
Total & 13 & 32 & 45 \\
\hline
\end{tabular}

El análisis de los actores principales y secundarios en las informaciones periodísticas es otro aspecto importante a considerar en el estudio de la representación de las mujeres parlamentarias en la prensa portuguesa. Así se observa que las diputadas han ocupado el protagonismo de actor principal en 32 ocasiones mientras que los diputados apenas han alcanzado 13 representaciones. (Cuadro ${ }^{\circ} 7$ ).

Cuadro $\mathrm{n}^{\mathrm{o}}$ 8-Total de actores (principales y secundarios) por Periódico y Partido Político

\begin{tabular}{cccccc}
\hline Partido Político & $\begin{array}{c}\text { Correio } \\
\text { da } \\
\text { Manhã }\end{array}$ & $\begin{array}{c}\text { Diário de } \\
\text { Notícias }\end{array}$ & Público & Expresso & Total \\
\hline BE & - & - & 1 & - & 1 \\
CDS/CDS-PP & - & 1 & 1 & - & 2 \\
PCP/CDU & - & - & 7 & 1 & 8 \\
PEV & - & 1 & 1 & - & 2 \\
PPD/PSD & - & 1 & 4 & 2 & 7 \\
PS & - & 2 & 5 & 2 & 9 \\
Independente & - & - & 1 & - & 1 \\
NA/ND & - & - & 2 & - & 2 \\
Total & - & 5 & 22 & 5 & 32 \\
\hline
\end{tabular}


Cuando analizamos a los actores principales y secundarios por publicación y partido político concluimos que los parlamentarios de PS ( 9 actores) y de PCP (8 actores) han tenido un mayor protagonismo en el Parlamento y han alcanzado más voz en los medios analizados, seguidos de los actores del PSD (7 actores). Teniendo en cuenta que el destino de la Ley de la Paridad estaba en sus manos, los tres partidos políticos no llegaron a un consenso por lo que se invalidó la aprobación de la ley. Y al analizar los actores por periódico observamos que Público se adelanta sobre los otros periódicos con un total de 22 actores.

\section{CONCLUSIONES}

-Aunque el primer ministro Antonio Guterres ha desarrollado una práctica política y un lenguaje centrados en el reconocimiento de la importancia de la participación política de las mujeres, llegando a ejercer un gran protagonismo respecto de esta cuestión, la ley de la cuotas ha sido rechazada por el Parlamento. El asunto no ha tenido el apoyo necesario por parte de los líderes parlamentarios que, además, no han tomado el asunto en serio en sus negociaciones.

-Sin embargo, durante el Guterrismo pasaron por el Parlamento un total de 61 diputadas. Se observa que estas diputadas son mayoritariamente representantes del Partido Socialista y se distancian mucho de la representación femenina del segundo mayor partido; el Partido Social Demócrata con 11 diputadas.

-Además este estudio demuestra que las diputadas tienen una significativa preparación académica puesto que el 87,09 por ciento tienen grados universitarios.

-Con respecto a la cobertura periodística comprobamos que ha sido escasa en los periódicos analizados: 14 informaciones publicadas en el diario Público y el resto casi han ignorado el tema. En la mayor parte de los periódicos el tema ha sido recogido en la sección de opinión y a través de sus respectivos comentadores políticos (6 en un total de 14 piezas). Esto demuestra que los medios de comunicación no han considerado relevante el asunto tratado en el Parlamento y por otro lado reflexiona sobre la división de opiniones en la sociedad.

-El periódico Público es, sin duda, el que más cobertura ha proporcionado a este debate parlamentario, tanto en número de informaciones, como en diversidad de géneros periodísticos así como en el protagonismo otorgado a las diputadas parlamentarias como actores en las informaciones periodísticas. Además, lo ha presentado de forma equilibrada escuchando a diversas fuentes y actualizando contenidos relacionados con la situación general de la mujer parlamentaria en Portugal.

-El análisis de los actores presentes en las piezas periodísticas representa un total de 32 mujeres frente a 13 hombres, lo que determina que algo parece estar cambiando en la representación de las diputadas parlamentarias y sobre todo, el cambio se percibe en su capacidad de intervención y autonomía en comparación con los líderes masculinos. A continuación notamos que no había consenso ni entre las diputadas ni tampoco entre las mujeres portuguesas.

-Las diferencias son especialmente notables con respecto al encuadre de los actores, ahora narrado de manera mucho más equilibrada escuchando las partes, evi- 
tando informaciones con cierta carga de opinión en encuadres cínicos o sarcásticos. Una situación que se ha dado doce años atrás en la cobertura periodística del "Parlamento Paritario" lo que ha significado un descalificativo en la iniciativa de las diputadas.

-Este debate parlamentario sobre la ley de la Paridad ha tenido, sin duda, una importancia política significativa. Por un lado, porque en el Guterrismo había sensibilidad por parte del Primer-Ministro y de la mayoría parlamentaria del Partido socialista. Por otro lado porque la divergencia no era sobre la cuestión de la igualdad en el acceso a los cargos políticos, pero si por la cuestión de la cuotas. Y esta divergencia no la tienen tampoco algunas de las diputadas Social Demócratas y Comunistas.

-De todas formas el debate sobre la necesidad de aplicar medidas de discriminación positiva era fundamental como alerta social para la realidad de un gran desequilibrio entre representación de género, no solo en el Parlamento sino en todos los cargos políticos en general.

-Los argumentos de las diputadas en el debate de la Ley de la Paridad eran representativos de las disertaciones que aún seguían siendo una realidad tanto al nivel político como en la sociedad en general. La ley ha sido rechazada en 2002, para ser finalmente aprobada en el año de 2006, en otro ciclo político con José Sócrates, también primer ministro socialista. La ley de la Paridad, aprobada en 2006, promueve que las listas para la Asamblea de la República, Parlamento Europeo, para los Ayuntamientos y autarquías locales debían estar compuestas de forma que garantizasen la representación mínima del 33\% de cada uno de los sexos.

-Sin embargo la representación de las diputadas en el Parlamento portugués ha sufrido un aumento lento y poco satisfactorio, porque los que tienen el poder de decisión sobre la selección y jerarquización de los la diputadas y de los diputados siguen siendo los hombres.

-La importancia de esta investigación, reside en el debate de la ley de las Cuotas, durante los 27 años analizados. Pasamos de una indiferencia en el Parlamento acerca de la representación de la mujer en la política a una preocupación relevante seguida de acciones y aprobaciones de leyes. En cuanto a la representación en los medios pasamos del silencio a una mayor visibilidad y protagonismo. De manera que nos parece de todo imprescindible que la investigación siga analizando los primeros diez años del siglo XIX y se concretice en una perspectiva comparativa entre la representación de la mujer parlamentaria en Portugal y España.

\section{BIBLIOGRAFÍA}

Abejón Mendonza, Paloma; SÁnCheZ Calero, M. Luisa; Vinuesa Tejero, M. Lourdes; CABRERA, Ana (2011). "Discurso e imagen de la presencia de la mujer en la política española". Articulo aprobado para publicación en Noviembre de 2011 en la Revista Faces de Eva. Portugal

AlmeidA, Ana Margarida Nunes de (1986). "Entre o dizer e o fazer: a construção da identidade feminina", Análise Social, vol. XXII (92-93), 1986-3. ${ }^{\circ}-4 .^{\circ}, 493-520$ 
Araúso, Clara (2005), Partidos políticos e género: mediações nas rotas de ingresso das mulheres na representação política, Revista de Sociologia Política, Curitiba, 24, p. 193-215, jun. 2005

Axford, B., Huggins, R., (2002), News, media and politics, London: Sage Publications.

BANDUCCI, Susan A. (2005) "Women, Elections and European News". Paper prepared for presentation at the ECPR Meetings, 8-10 September, 2005. Budapest, Hungary.

Boorstin, D. (1961). The Image: Or What Happened to the American Dream? New York: Atheneum.

Boorstin, D. (1971) "From news-gathering to news-making: A Flood of pseudoevents". In Schramm e Roberts (eds). The Process and Effects of Mass Communication. Urbana, Ill: Universiy of Illinois Press.

CABO, Rosa (2002). Democracia Paritaria y sujeto político feminista, Anales de la Cátedra de Francisco Suarez. 36, 2002. 29-44

García, A.; Gómez Escalonilla, G; Rodríguez, R, Santín, M. y Torregrosa, J. F. (2008): Una aproximación a la representación y percepción de la mujer política. IBERCOM: Grupo de Trabajo: Comunicación y género, IX Congreso.

Galligan, Yvonne (2006). "Bringing Womwn in: Global Strategies for Gender Parity in Political Representation". In U. MD. L. J. Race, Religion, Gender \&Class, Vol, 6:319

Grossi, Míriam Pillar, Miguel, Sônia Malheiros (2001). Transformando a diferença; As mulheres na política. Estudos Feministas, $2^{\circ}$ semestre 2001

Freire, André, VIEGAS, José Manuel, SEICEIRA, Filipa, (2009), Representação Política em Portugal, Lisboa:Sextante Editora

KroOK, Mona Lena (2006). Reformin Representation: The Diffusion of Candidate Gender Quotas Woldwide, in Politics \& Gender, 2, 303-327, Published by Cambridge University Press

Lovecy, Jill, (2002). Gender Mainstreaming and the Framing of Women's Rights in Europe: the Contribution of the Council of Europe, in Feminist Legal Studies 10: 271-283.

Lovenduski, Joni and Karam Azza (2002), Women in Parliament: Making a Difference, International IDEA, Stockholm (http://www.idea.int)

McCulloch, John E.S. (2009), Women in Parliament: Attaining the Ideal Australasian Parliamentary Review, Autumn 2009, Vol. 24(1), 122-36.

NORRIS, Pippa (2000). "Breaking the Barriers:Positive Discrimination Policies for Women". In Eds. Jyette Klausen and Charles S. Maier. Has Liberalism Failed Women? Parity, Quotas and Political Representation. NY: St Martin's Press.

NORRIS, Pippa and LovENDUSKI, Jony, (1986). Women candidates for Parliament: transforming the Agenda? British Journal of Political Science, Vol 19, nº 1 (Jan. 1989), 106-115

PHILliPS, Anne, (1998). "Democracy and Representation: or, Why Should it Matter Who our Representatives Are?", in Phillips Anne Ed. Feminism and Politics. Oxford University Press

Ross, Karen, (2003). Women Politicians and Malestream Media: a game of two sides. Centre for Advancement of Women in Politics. Ocsasional paper, 1.

SANTOS, Helena, AmÂNCIO, Lígia (2004). Uma perspectiva psicossociológica da controvérsia em torno das acções positivas: $\mathrm{O}$ género em contexto político Actas dos ateliers do $V^{o}$ Congresso Português de Sociologia Sociedades Contemporâneas: Reflexividade e Acção Atelier: Género 
SGIER, Lea 2003. Political Representation and Gender Quotas. Paper for delivery at ECPR Joint Sessions, Edinburgh, UK

SiLva, Maria Regina Tavares da (1994). Democracia Paritária um conceito novo ou um novo olhar sobre a democracia Lisboa, CIDM, 1994

SilveIRINHA, Maria João, (2008). O discurso feminista e os estudos dos media: em busca da ligação necessária, Disponível em: http://www.bocc.ubi.pt/pag/silveirinha-mariajoao-discurso.pdf

SilveIRINHA, Maria João, (2009), De como tanto mudou e como tanto ficou na mesma, Media \& Jornalismo, . $^{\circ} 15$, vol, $8, \mathrm{n}^{\circ}{ }^{\circ} 2$ Outono/Inverno, 7-11

SilveIRINHA, Maria João, PEIXINHO, Ana Teresa (2008), The Law of Life and the Law of Quotas, Artigo no publicado sobre el Proyecto "Mujeres y Esfera pública: el papel de los medios en áreas críticas de la Plataforma de Pekín, apoyado por FCT

TAVARES, Manuela (2003), "Políticas neoliberais e participação política e social das mulheres". Fórum Social Português. Conferência:"As políticas neoliberais e a degradação da democracia". 7 de Junho de 2003

TAVRes, Manuela (2011). Feminismos: percursos e desafios (1947-2007). Lisboa: Texto Editores

ViCENTE, Ana (1997). 20 anos ao serviço da Igualdade, Presidência do Conselho de Ministros. Alto-comissário para as Questões da Igualdade e da Família, Comissão para a Igualdade e para os Direitos da Mulher, Lisboa

VilATOD I PRESAS: "La imagen de las mujeres políticas en los medios de comunicación”.En VV.AA.(2008): Mujeres, politica y medios de comunicación. Homenaje a Clara Campoamor. Sevilla: Fundación Audiovisual de Andalucía.

Vinuesa Tejero,I; Abejón Mendoza, P y SÁnchez Calero, Ma L. (2011). "Mujeres y política: un binomio con baja representación" En Revista Ámbitos No 20.

Zetterberg, Pär (2009). Do Gender Quotas Foster Women's Political Engagement? In Political Research Quarterly, Vol. 62, Number 4, 715-730. University of Utah. $\mathrm{http} /$ prq.sagepub.com 


\title{
RESUMEN
}

Esta investigación analiza las estrategias de visibilidad que han alcanzado en la prensa portuguesa las mujeres parlamentarias durante los tres ciclos políticos de democracia correspondiente al periodo que va desde 1976 al año 2002. El objeto de estudio es, por tanto, doble: por un lado, determinar el grado de representación que alcanzan las mujeres en el parlamento, la cuota alcanzada en cada grupo parlamentario y el perfil trazado de las diputadas en cada ciclo político Y, por el otro, la representación que la prensa portuguesa hace de dichas mujeres políticas a través de los estereotipos que han conformado su agenda pública.

Los resultados obtenidos proceden de una investigación enmarcada en un proyecto de investigación del Centro de Investigación Media e Jornalismo (CIMJ) de la Universidad Nova de Lisboa I+D (PTDC/CCI_COM 7102393/2008).

Palabras clave: género, diputadas parlamentarias, política, cuotas, ley de la Paridad, periodismo

\begin{abstract}
This research analyzes the strategies of visibility achieved in the Portuguese press by female parliamentarians during the three political cycles of democracy for the period ranging from 1976 to 2002 . The object of study is therefore twofold: on the one hand, the extent of representation that women reach in Parliament, the share achieved in each parliamentary group and the layout of the profile of the members in each political cycle and, on the other, the study of the representation that the Portuguese press makes of these politician women through the stereotypes that have shaped the public agenda. The results come from an analysis framed in a research project of the Centre for research Media \& Jornalismo (CIMJ) of the University Nova of Lisbon d (PTDC/CCI_COM 7102393/2008).
\end{abstract}

Keywords: gender, politics, quotas, parliamentary deputies, law of parity, journalism

\section{RÉSUMÉ}

Cette recherche analyse les stratégies de visibilité, obtenuEs dans les presse portugaise par les femmes parlementaires pendant les trois cycles politiques de la démocratie, dans la période entre 1976 et 2002. L'objet d'étude est donc double : d'une part, l'étendue de la représentation qui atteignent les femmes au Parlement, la présentation de profil et le groupe parlementaire des membres dans chaque cycle politique et, d'autre part, la représentation que la presse portugaise fait des femmes politiques à travers les stéréotypes qui ont façonné son agenda. Les résultats proviennent d'une recherche formulée dans un projet du Centre de recherche Médias \& Jornalismo (CIMJ) de l'Université Nova de Lisbonne d (PTDC/CCI_COM 7102393/2008).

Mots clé: sexe, politique, quotas, députés parlementaires, Loi de la parité, journalisme 